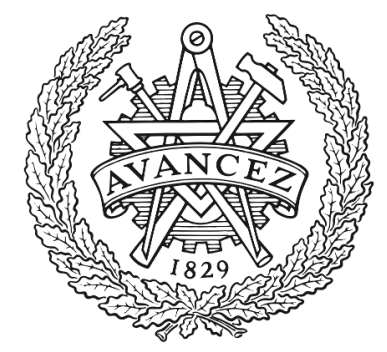

CHALMERS

UNIVERSITY OF TECHNOLOGY

\title{
Accurate chemical analysis of graphene-based materials using X-ray photoelectron spectroscopy
}

Downloaded from: https://research.chalmers.se, 2023-04-26 11:37 UTC

Citation for the original published paper (version of record):

Kovtun, A., Jones, D., Dell'Elce, S. et al (2019). Accurate chemical analysis of graphene-based materials using X-ray photoelectron spectroscopy. Carbon, 143: 268-275.

http://dx.doi.org/10.1016/j.carbon.2018.11.012

N.B. When citing this work, cite the original published paper. 


\section{Accurate chemical analysis of graphene-based materials using X-ray photoelectron spectroscopy}

Alessandro Kovtun ${ }^{\text {a }}$, Derek Jones ${ }^{\text {a }}$, Simone Dell'Elce ${ }^{\text {a }}$, Emanuele Treossi ${ }^{\text {a }}$, Andrea Liscio ${ }^{\text {a, b, * }}$, Vincenzo Palermo ${ }^{\text {a, c }}$

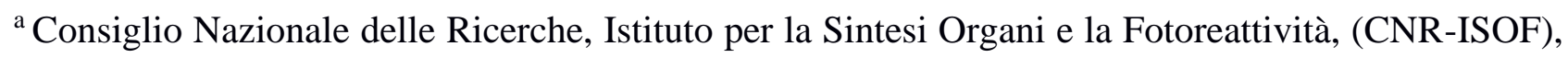
Via Gobetti 101, 40129 Bologna, Italy

${ }^{\mathrm{b}}$ Consiglio Nazionale delle Ricerche, Istituto per la Microelettronica e Microsistemi, (CNR-IMM), via del Fosso del Cavaliere 100, 00133 Roma, Italy

${ }^{\mathrm{c} C h a l m e r s ~ U n i v e r s i t y ~ o f ~ T e c h n o l o g y, ~ D e p a r t m e n t ~ o f ~ I n d u s t r i a l ~ a n d ~ M a t e r i a l s ~ S c i e n c e, ~ H o ̈ r s a l s v a ̈ g e n ~}$ 7B, SE-412 96 Gothenburg, Sweden

* Corresponding author: email address: andrea.liscio@artov.imm.cnr.it (Andrea Liscio) 


\section{Abstract}

A simple, fast and general protocol for quantitative analysis of X-ray photoelectron spectroscopy (XPS) data provides accurate estimations of chemical species in graphene and related materials (GRMs). XPS data are commonly used to estimate the quality of and defects in graphene and graphene oxide (GO), by comparing carbon and oxygen 1s XPS peaks, obtaining an O/C ratio. This approach, however, cannot be used in the presence of extraneous oxygen contamination.

The protocol, based on quantitative line-shape analysis of C 1s signals, uses asymmetric pseudoVoigt line-shapes (APV), in contrast to Gaussian-based approaches conventionally used in fitting XPS spectra, thus allowing better accuracy in quantifying $\mathrm{C} 1 \mathrm{~s}$ contributions from graphitic carbon $\left(\mathrm{sp}^{2}\right)$, defects ( $\mathrm{sp}^{3}$ carbon), carbons bonded to hydroxyl and epoxy groups, and from carbonyl and carboxyl groups. The APV protocol was evaluated on GRMs with $\mathrm{O} / \mathrm{C}$ ratios ranging from 0.02 to 0.30 with film thicknesses from monolayers to bulk-like (>30nm) layers and also applied to previously published data, showing better results compared to those from conventional XPS fitting protocols.

Based uniquely on C 1s data, the APV protocol can quantify $\mathrm{O} / \mathrm{C}$ ratio and the presence of specific functional groups in GRMs even on $\mathrm{SiO}_{\mathrm{x}}$, substrates, or in samples containing water.

Keywords: C 1s, Graphene, Graphite, Oxidation degree, XPS, Quantitative analysis. 


\section{Introduction}

Graphene and related materials (GRMs) have a wide range of different chemical, electrical and electronic properties which render them useful for technological applications in composites, energy storage, sensing, multifunctional materials, etc. [1-3]. A major issue hindering large-scale application of such materials on an industrial scale is related to quality control and metrology: industrial endusers are often confused by the wide range of commercially available graphene products, often with questionable claims of outstanding quality and properties.

There is thus an urgent need to develop a graphene metrology based on standard definitions and techniques, allowing valid comparisons between different materials [4, 5]. Unfortunately, characterization of 2D materials such as graphene is not currently based on standard techniques such as those used for quality control of 1D polymers.

An important step in this direction has recently been taken by Bianco et al. proposing a classification of GRMs based on three parameters (fig. 1): average lateral size, average number of layers and degree of oxidation (O/C ratio) [6,7]. We have previously addressed the task of measuring lateral sizes of 2D nanosheets with high throughput and sound statistics, using automated image processing [2, 8]. Here, the focus is on the characterization of the chemical composition of GRMs and, in particular, on their oxygen content (i.e., the $X$ axis in fig. 1). This property is commonly expressed as the overall $\mathrm{O} / \mathrm{C}$ ratio of oxygen and carbon atoms present in the material.

The main technique used to characterize GRMs is Raman spectroscopy, which can evaluate the defectivity of the honeycomb graphene lattice using vibrational phonons. This technique is ideal for comparing high-quality GRMs, such as monolayers grown by chemical vapour deposition (CVD) for electronics applications. However, it is less effective for characterizing GRMs obtained by graphite exfoliation, often consisting of small platelets, with high defectivity, where oxygen-containing defects are intentionally added to enhance solubility and processability in composites. The size of the D Raman peak, commonly used to estimate graphene quality, increases with the number of defects, but then decreases or even disappears for highly defective carbon-based materials [9].

An alternative technique for obtaining detailed information on the chemical composition of GRMs is X-rays Photoemission Spectroscopy (XPS) [10]. This is a quantitative and reliable technique using $\mathrm{X}$-rays to remove electrons from the $\mathrm{C} 1 \mathrm{~s}$ and $\mathrm{O} 1 \mathrm{~s}$ levels of graphene and GRMs. The energies of the emitted electrons depend on the atoms present and thus the chemical composition of the material. This allows the quantification of elemental composition in the parts-per-thousand range, as well as the nature of the chemical bonds. XPS can thus provide a measure of the number of defects through 
the $\mathrm{O} / \mathrm{C}$ ratio, quantify the different types of carbon functionalities present, indicate the formation of chemical bonds, and evaluate the physisorption of molecules [11, 12]. The importance of this quantification is crucial in order to correlate chemical properties of GRMs with their performance, for example, in permeability [5], water purification [13] or bio-sensing [14]. Although XPS is an ideal technique for characterizing GRMs, results available in the literature using this technique are often rather incoherent.

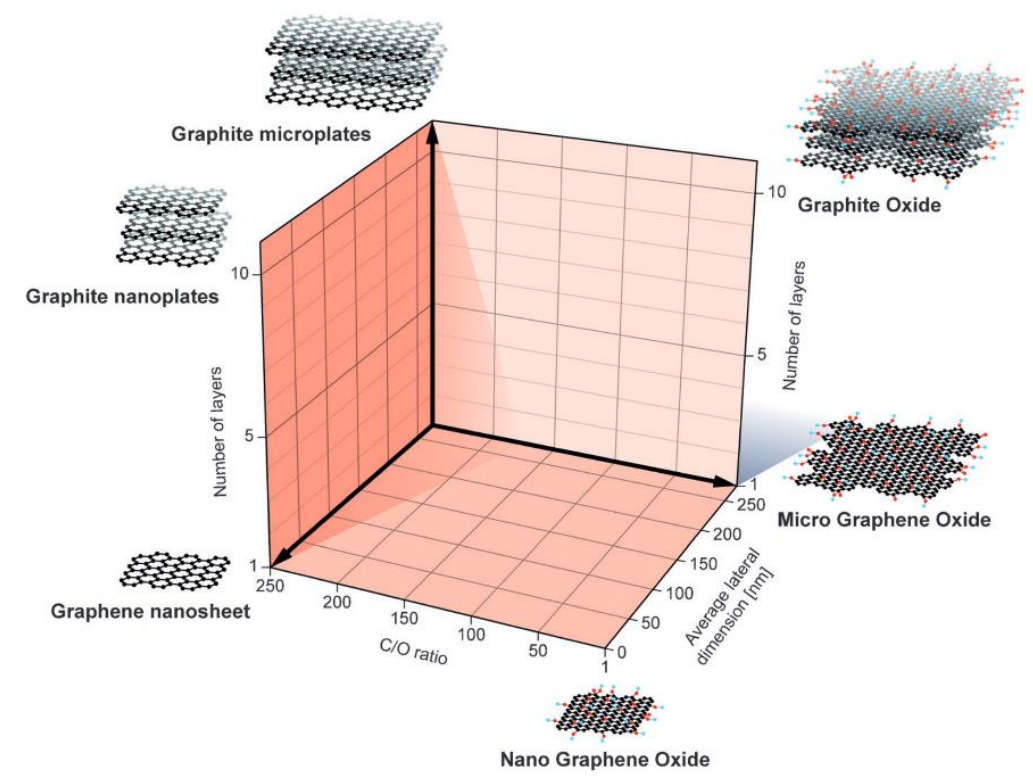

Fig. 1. Classification grid for the categorization of different graphene types according to three fundamental GRM properties: number of graphene layers, average lateral dimension, and atomic carbon/oxygen ratio. From ref. [6], with permission.

The O/C ratio is the most widely used and cited parameter in quantitative XPS analysis of GRMs, and can be calculated using two methods:

1) AREA METHOD, which compares the overall areas of $\mathrm{O} 1 \mathrm{~s}$ and $\mathrm{C} 1 \mathrm{~s}$ XPS signals to give the oxygen/carbon atomic ratio $\left(\mathrm{O} / \mathrm{C}_{\text {area }}\right)$;

2) FITTING METHOD, based on the deconvolution of the $\mathrm{C} 1 \mathrm{~s}$ signal into its various contributions: graphitic carbon $\left(\mathrm{sp}^{2}\right)$, defects (mainly $\mathrm{sp}^{3}$ carbon), carbons bonded to hydroxyl and epoxy groups, and those from carbonyl and carboxyl groups. The $\mathrm{O} / \mathrm{C}_{\text {fit }}$ is then calculated using these $\mathrm{C} 1 \mathrm{~s}$ signals, without using the oxygen peak.

The area method is the more direct and accurate method with no systematic errors, but can only be used where there are no external oxygen signal sources. To prevent artefacts influencing the $\mathrm{O} 1 \mathrm{~s}$ peak, all measurements have to be performed on GRMs deposited on oxygen-free substrates (e.g. clean $\mathrm{Au}$ ) or on "thick" GRM samples (> $15 \mathrm{~nm}$ ). The presence of oxygen, for example, prohibits a 
direct calculation of $\mathrm{O} / \mathrm{C}_{\text {area }}$ on $\mathrm{SiO}_{2}$, the main substrate of interest for electronics applications. In general, the interpretation of $\mathrm{O} 1 \mathrm{~s}$ spectra is not straightforward because O1s peaks tend to be broad, with multiple overlapping components, and preventing a clear distinction between the contribution of the substrate and that of the deposited GRM. For this reason, we focussed our efforts to the quantitative analysis of $\mathrm{C} 1 \mathrm{~s}$ surveys.

The fitting method, on the other hand, can be applied to all substrates and sample thicknesses, but usually shows systematic and intrinsic errors due to the fitting procedure used: the various $\mathrm{C} 1 \mathrm{~s}$ signals are usually fitted with symmetric Voigt curves, based on a convolution of Gaussian and Lorentzian curves [15]. To compare the accuracy of these two methods, we analysed the data from a number of published XPS studies on various GRMs, which calculated the $\mathrm{O} / \mathrm{C}_{\text {area }}$ (measuring the area of the peaks) and the $\mathrm{O} / \mathrm{C}_{\mathrm{fit}}$, fitting the $\mathrm{C} 1 \mathrm{~s}$ peak with multiple symmetric Voigt curves.

These XPS analyses include those by Stobinski et al. [16], Mattevi et al. [17], Poh et al. [18], Jankovsky et al. [19] and Perrozzi et al. [20]. The XPS of Chemical Vapor Deposited Graphene (CVD-G) was reported by Ray et al. [21] and the XPS of Multiwall Carbon Nanotubes (MWCNT) by An et al. [22].

The articles cited above report both $\mathrm{C}$ 1s and O 1s XPS signals and the O/C values calculated with the area method. These published $\mathrm{O} / \mathrm{C}$ values were then compared with the values we calculated by deconvoluted C1s signals, as reported in such publications.

Figure 2 shows the correlation plot of the O/C values obtained from the published data using the two methods. The experimental details and data are reported in the Supporting Information (SI). Ideally, the estimated $\mathrm{O} / \mathrm{C}$ ratios should not depend on the method used $\left(\mathrm{O} / \mathrm{C}_{\mathrm{fit}}=\mathrm{O} / \mathrm{C}_{\text {area }}\right)$ and should lie on the bisector line of figure 2 .

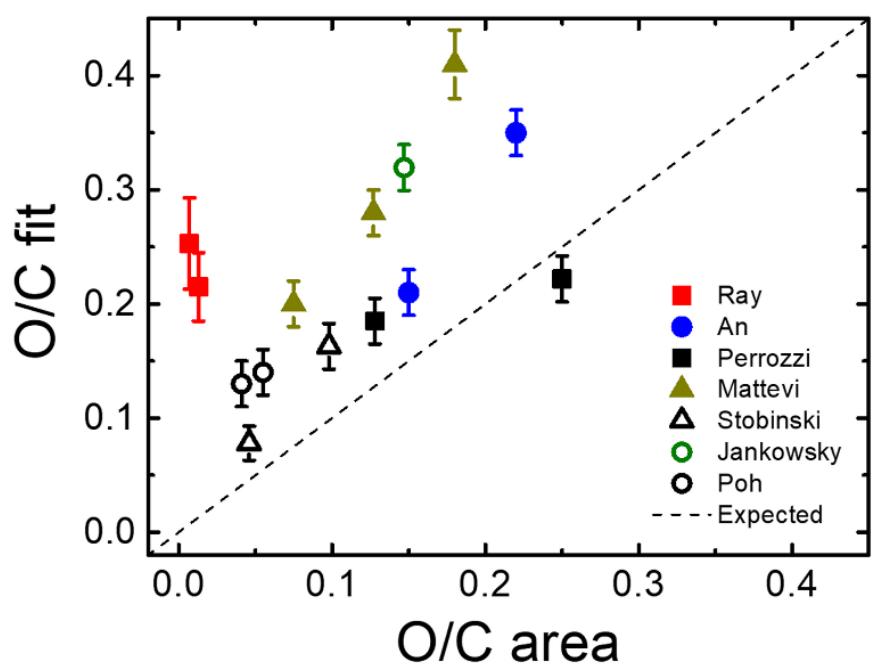

Fig. 2. Correlation plot of the oxygen/carbon ratio calculated using the area method $\left(\mathrm{O} / \mathrm{C}_{\text {area }}\right)$ and the fit method $\left(\mathrm{O} / \mathrm{C}_{\mathrm{fit}}\right)$ in selected published works, showing that the two methods do not correlate. Data 
from: Ray et al. [21]; An et al. [22]; Perrozzi et al. [20]; Mattevi et al.[17]; Stobinski et al. [16]; Jankowski et al. [19]; Poh et al. [18].

Figure 2 shows clearly that the two measurements are not coherent with each other, and that there is a systematic overestimation of the $\mathrm{O} / \mathrm{C}_{\text {fit }}$ values in the published data. In fact, estimation of $\mathrm{O} / \mathrm{C}$ ratios of GRMs deposited on oxygen-rich substrates such as $\mathrm{SiO}_{\mathrm{x}}$, mica, glass or steel are likely to be inaccurate [23] suggesting that studies presenting a chemical analysis of graphene-based devices insitu [24-28] could be affected by an intrinsic overestimation of the oxygen content.

As previously suggested by Yumitori [29], such overestimation can be due to an incorrect fitting procedure. In fact, the $\mathrm{C} 1 \mathrm{~s}$ peak is conventionally deconvoluted using symmetric Voigt curves for all the $\mathrm{C} 1 \mathrm{~s}$ peaks present, including the aromatic $\mathrm{C} 1 \mathrm{~s}$ component from $\mathrm{sp}^{2}$ carbon atoms which is known to be asymmetric [30]. This is an intrinsic effect due to the interaction of the positive inner core hole with electrons in the valence band. Thus, an asymmetric function is more appropriate for describing the C 1s XPS peak of aromatic carbons [31, 32].

The use of a line-shape compatible with the known XPS peak asymmetries should, therefore, reduce such over-estimations of $\mathrm{O} / \mathrm{C}_{\text {fit }}$ ratios from XPS data.

To verify this hypothesis, we thus prepared several series of GRM samples with a wide range of O/C ratios, ranging from 0.02 to 0.30 including graphite, CVD graphene, electrochemically exfoliated graphite and reduced GO, which were then characterised by XPS. For each sample, the O/C ratio was calculated with:

1) AREA METHOD, using the $\mathrm{C} 1 \mathrm{~s}$ and $\mathrm{O}$ 1s peaks.

2a) Symmetric Fitting protocol using conventional symmetric Voigt functions as in the literature;

2b) Asymmetric Fitting protocol using asymmetric pseudo-Voigt (APV) functions, taking into account the semi-metallic behaviour of $\mathrm{sp}^{2}$ carbons.

The contributions of all the other functional groups and the $\mathrm{sp}^{3} \mathrm{C} 1 \mathrm{~s}$ signals were fitted using standard symmetric curves.

Once validated, this comparative analysis on samples prepared for this purpose, it was applied to the previously published experimental data cited above which were digitalised and fitted with the APV method.

\section{Experimental methods}

\subsection{Sample preparation}


The samples we prepared and analysed with XPS were:

a) Highly Oriented Pyrolytic Graphite (HOPG) (grade ZYH, Advanced Ceramics, Cleveland USA).

b) Graphene Oxide (GO) films produced via a modified Hummers method [20, 33, 34]. Thin films of GO were produced by spin-coating a GO water suspension on cleaned silicon (see SI) and their thickness measured by AFM. The thickness of each single layer of GO was $1.0 \pm 0.1 \mathrm{~nm}$, the sample thickness then being given as the number of layers [35].

c) Reduced Graphene Oxide (RGO) films were prepared by thermal annealing of GO samples in high vacuum ( $\mathrm{HV}, 10^{-7} \mathrm{mbar}$ ), at different annealing temperatures (from $160^{\circ} \mathrm{C}$ to $900^{\circ} \mathrm{C}$ ). Upon reduction to RGO, the layer thickness decreased to $0.4 \pm 0.1 \mathrm{~nm}$ in agreement with previous results [35]. Thickness calibration measurements as a function of the number of consecutive spincoatings are reported in the SI.

d) 3-dimensional graphene structures grown by Chemical Vapor Deposition (CVD-G) on selfstanding nickel foams [36]. Thus, our CVD-G was grown on nickel foams, producing 3dimensional structures which could be easily handled. CVD produces graphene of high quality, although not quite as good as mechanical exfoliation using adhesive tape [37]. The nickel foam was then acid-etched, leaving all-carbon, self-standing structures of pure CVD-G, without residual PMMA contamination. CVD-G transferred on gold substrate was not used as testing due to significant contamination caused by polymers used for sample transfer, i.e. poly(methylmethacrylate) (PMMA), poly(lactic acid) (PLA), poly(phthalaldehyde) (PPA), and poly(bisphenol A carbonate) (PC).

All of them are carbon/oxygen polymers and their presence on the surface can strongly affect the measurement and the calculation of the O/C ratio of GRM.

e) Electrochemically-Exfoliated Graphene Oxide (EGO), prepared as previously described [38] to produce nanosheets with tunable degrees of oxidation. Macroscopically thick membranes (thickness $\approx 100 \mathrm{~nm}$, diameter $\approx 4 \mathrm{~cm}$ ) were prepared by filtering the EGO-water suspension. The membrane obtained was deposited on $\mathrm{SiO}_{\mathrm{x}}$ and dried in the UHV chamber at $200^{\circ} \mathrm{C}$ for $1 \mathrm{~h}$ (or until the Reduced Gas Analyser showed no residual water). The XPS spectrum of EGO prepared in this way was comparable to previously published data [14].

\subsection{X-ray Photoelectron Spectroscopy}

High-resolution XPS spectra of all samples were obtained using a Phoibos 100 hemispherical energy analyser (Specs $\mathrm{GmbH}$, Berlin, Germany) and $\mathrm{Mg} \mathrm{K \alpha}$ radiation $(\hbar \omega=1253.6 \mathrm{eV}$; power $=125 \mathrm{~W})$ in 
constant analyser energy (CAE) mode, with analyser pass energies of $10 \mathrm{eV}$. The overall resolution of $0.9 \mathrm{eV}$ was measured and spectra calibrated using the $\mathrm{Ag} 3 \mathrm{~d}_{5 / 2}(368.3 \mathrm{eV})$ and $\mathrm{Au} 4 \mathrm{f}_{7 / 2}(84.0 \mathrm{eV})$ signals from freshly $\mathrm{Ar}^{+}$sputtered samples. Base pressure in the analysis chamber during analysis was $5 \times 10^{-10}$ mbar.

Data analysis and fitting were performed with CasaXPS software ${ }^{1}$, after Shirley background subtraction [39].

Peak positions of the non-equivalent carbon species, based on literature data, were: aromatic carbon $\left(\mathrm{C}-\mathrm{C} \mathrm{sp}{ }^{2}, 284.4 \mathrm{eV}\right)$, aliphatic carbon $\left(\mathrm{C}-\mathrm{C} \mathrm{sp}{ }^{3}, 285.0 \mathrm{eV}\right)$, hydroxyl (C-OH, $\left.285.7 \mathrm{eV}\right)$, epoxy (C-O$\mathrm{C}, 286.7 \mathrm{eV})$, carbonyl $(\mathrm{C}=\mathrm{O}, 288.0 \mathrm{eV})$ and carboxyl $(\mathrm{O}-\mathrm{C}=\mathrm{O}, 290.1 \mathrm{eV})$ [10, 16, 40-42]. Figure S1 compares the various $\mathrm{C}$ 1s chemical shifts, as reported in the literature. Further secondary peaks corresponding to plasmon/shake-up contributions are centred at $+6.4 \mathrm{eV}$ and $+10.1 \mathrm{eV}$ with respect to the main $\mathrm{sp}^{2}$ peak [43]. Analysis of the previously published XPS data was performed by extracting the $\mathrm{C}$ 1s spectrum from each article using the Matlab Grabit routine ${ }^{2}$. More detail on instrumental configuration and data analysis is available $[14,34]$.

\subsection{Atomic Force Microscopy (AFM)}

AFM was used to measure the film thickness using a Bruker MultiMode 8, with probe cantilevers model RTESPA-300 (material: 0.01-0.025 $\Omega \mathrm{cm}$ Sb-(n)-doped Si, fo: $300 \mathrm{kHz}, \mathrm{k}$ : 40 N/m) working in the tapping mode. We report the film thickness as the number of layers $\mathrm{N}$, following a previously reported procedure [35], since the thickness of single layers of GO and RGO changes from 1.0 to 0.4 $\mathrm{nm}$ as a function of the annealing temperature. For all the RGO devices, we calculated the equivalent number of layers as the ratio between the measured thickness of the GO (i.e. before reduction) film and the known thickness of the single GO sheet $(1 \mathrm{~nm})$.

\section{Results and discussion}

As mentioned in the introduction, peaks deriving from $\mathrm{sp}^{2}$ and $\mathrm{sp}^{3}$ carbons have different $\mathrm{C}$ 1s signals when studied by XPS.

Defects in the graphene lattice due to $\mathrm{sp}^{3}$ carbon atoms and those in covalently linked functional groups can be described by symmetric Voigt curves (V). Such curves have no analytical form;

\footnotetext{
${ }^{1}$ CasaXPS, www.casaxps.com

${ }^{2}$ Matlab it.mathworks.com
} 
although a symmetric Voigt curve can be modelled by a convolution of Gaussian (G) and Lorentzian (L) curves: $V=(G * L)$ (see Eqn. 1 below). The GL function is a good analytical approximation of a Voigt function, and is known as Pseudo-Voigt [44]; the G curve describes the contribution of the experimental setup (noise, energy/angular resolution, etc.), the L curve describes the intrinsic atomic spectral line [45]. Other initial- and final-state effects [46] can be neglected at this resolution [47]. We thus fitted the symmetric peaks using the symmetric function GL(p), the product of G and L curves, combined together using a weighting factor $p$ (see SI).

$V \approx G L\left(x: \sigma, \gamma, x_{0}, p\right)=\frac{1}{\pi \gamma \sigma \sqrt{2 \pi}} \cdot \frac{e^{-4 \cdot \ln 2 \cdot\left(1-\frac{p}{100}\right) \cdot \frac{\left(x-x_{0}\right)^{2}}{\sigma^{2}}}}{\left(1+4 \cdot \frac{p}{100} \cdot \frac{\left(x-x_{0}\right)^{2}}{\gamma^{2}}\right)}$

The variable $x$ corresponds, for XPS profiles, to the binding energy (B.E.). $\mathrm{x}_{0}$ corresponds to the peak position, while $\sigma$ and $\gamma$ are the widths of the Gaussian and the Lorentzian peaks, respectively.

For our analysis $\mathrm{p}=50 \%$ was used, with a full-width-half-maximum (FWHM) between 1.2 and 1.4 $\mathrm{eV}[31]$.

In graphite and high-quality graphene, on the other hand, all carbon atoms are $\mathrm{sp}^{2}$ hybridized, with a consequent asymmetric C 1s signal, due to the density of states (DOS) near the Fermi edge and to many-body screening effects [31, 32, 48, 49].

One of the most common curve used to reproduce the asymmetry of the XPS peak is the well-known Doniach-Sunjic (DS) [50] function. However, since its integral diverges is not suitable for multi-peak fitting.

We thus fitted the asymmetric C $1 \mathrm{~s} \mathrm{sp}^{2}$ signal using an asymmetric pseudo-Voigt (APV) function. The function is a numeric convolution of an asymmetric Lorentzian curve, centered at $x_{0}$, and a Gaussian curve $(\mathrm{G})$ :

$A P V=L F\left(x: \gamma, x_{0}, \alpha, \beta, w, m\right)=G\left(x: \sigma(m), x_{0}\right) * \begin{cases}{\left[L\left(x: \gamma, w, x_{0}\right)\right]^{\alpha}} & x \leq x_{0} \\ {\left[L\left(x: \gamma, w, x_{0}\right)\right]^{\beta}} & x>x_{0}\end{cases}$

here, $\alpha$ and $\beta$ are the damping parameters of the Lorentzian functions, $w$ is the relative weight of the Lorentzian curve and $m$ is the proportional factor between $\sigma$ and $\gamma\left(\sigma=\frac{m}{500} \cdot \gamma\right)$. The two formulae and the parameters used are described in detail in SI. The specific APV we use (LF, eq 2) is optimized for multi-peak fitting procedures and describes well the asymmetric peak shape [44]. We obtained the correct parameters for the APV function by calibration of the $\mathrm{C} 1 \mathrm{~s} \mathrm{sp}^{2}$ signal of freshly-cleaved HOPG (100\% $\mathrm{sp}^{2}$ carbon). For this sample, the FWHM was $0.82 \pm 0.02 \mathrm{eV}$ and the asymmetry parameter was 0.14 [49] (see SI).

All the $\mathrm{sp}^{2}$ components of the $\mathrm{C} 1 \mathrm{~s}$ spectra presented in this paper were fitted by setting the asymmetry parameter to 0.14 . This corresponded to fix the values of $\alpha, \beta$ and $m$ while the area is a free parameter 
and the FWHM values were ranged between 0.8 and $1.3 \mathrm{eV}$, due to the energy resolution of the spectrometer.

All the other $\mathrm{C}$ components were fitted by Gaussian peaks with the overall $\mathrm{C}$ 1s envelope, setting the peak positions at fixed position - with respect to the $\mathrm{sp}^{2}$ peak (1-position parameter). Similarly to the case of aromatic peak, areas and FWHMs of all the C contributions were free parameters where the latter were optimized within a constrained range (1.2-1.4 eV).

\subsection{Calculation of the $\mathrm{O} / \mathrm{C}$ ratio}

We first calculated the $\mathrm{O} / \mathrm{C}_{\text {area }}$ from the ratio between the areas of the oxygen and carbon $1 \mathrm{~s}$ signals, taking into account the Relative Sensitivity Factors (RSF) given by photoemission cross-sections and analyser transmission:

$O / C_{\text {area }}=\frac{A_{O 1 S}}{A_{C 1 S}} \cdot \frac{R S F_{C 1 S}}{R S F_{O 1 S}}$

Although this is the most accurate and effective procedure, it can only be applied in the case of oxygen-free substrates or bulk samples where the thickness is much larger than the photoelectron mean free path in the sample. In all the other cases, the oxygen present in the substrate will contribute to the spectrum, thus giving an overestimation of the $\mathrm{O} / \mathrm{C}$ ratio. Thus, this procedure cannot be used when graphene is supported on a common substrate such as silicon, due to the native oxide layer.

The $\mathrm{O} / \mathrm{C}$ ratio using the fitting protocols was then calculated, by deconvolution of the $\mathrm{C}$ 1s signal, which is the sum of the various $\mathrm{C} 1 \mathrm{~s}$ peaks of the $\mathrm{C}$-containing chemical species present. In this case, both the photoemission cross-sections and analyser transmission terms do not change: RSFs are constant and the total area $\left(\mathrm{A}_{\text {tot }}\right)$ of all the $\mathrm{C}$ 1s peaks is directly proportional to the number of carbon atoms present. The area of each single peak $\left(\mathrm{A}_{\mathrm{i}}\right)$ is proportional to the number of carbon atoms in the relative functional group. In this way, it is possible to estimate the overall $\mathrm{O} / \mathrm{C}$ ratio given by the contribution of the different species present: hydroxyl (1 to 1), epoxy (1 to 2), carbonyl (1 to 1 ) and carboxyl (2 to 1) according to the formula:

$O / C_{f i t}=\frac{A_{C-O H}+A_{C=O}+\frac{1}{2} \cdot A_{C-O-C}+2 \cdot A_{O-C=O}}{A_{\text {tot }}}$

This approach does not require monitoring of the oxygen photoemission peak, removing any artefact due to spurious oxygen presence in the substrate or, more in general, contribution of oxygen not chemically bounded with carbon atoms.. 
The C 1s sp ${ }^{2}$ peak was fitted using two protocols: (a) with the conventional symmetric Voigt function (eq. 1) and (b) APV using an asymmetric line shape (eq. 2). All the other components were fitted with symmetric Pseudo-Voigt curves, the peak positions remaining fixed for both protocols.

To evaluate how much the substrate contributes to the $\mathrm{O} / \mathrm{C}_{\text {fit }}$ signal we used, as an ideal test system, layers of RGO prepared with varying thickness on an $\mathrm{SiO}_{2}$ substrate [35]. Sample thickness was measured using AFM and also by monitoring the Si $2 p$ peak of the silicon substrate. The Si $2 p$ contribution became negligible for RGO thicknesses above $14 \mathrm{~nm}$, equivalent to $\approx 35$ layers given a thickness of $0.4 \mathrm{~nm}$ for individual layers, in agreement with previous results (see SI). Thus, for all successive measurements we used GO and RGO sample thicknesses above $14 \mathrm{~nm}$.

The thickness of CVD-G grown on nickel had no substrate contribution, given that the nickel was removed by etching before measurements [36]. The EGO sample membranes also had no substrate contribution, being several micrometers thick.

Figure 3 shows, as an example, C 1s spectra of CVD-G fitted with both protocols. In figures 3 and following, all the XPS spectra show the main functional groups labelled with different colours (the $\mathrm{sp}^{2}$ peaks are shown in red).

In general, both procedures gave a good fit of the experimental data (all the calculated reduced chi square values $\tilde{\chi}^{2}$ [51] are lower than 3 , as reported in table S6). In most of cases, the proposed APV protocol gave on average lower $\tilde{\chi}^{2}$ values than conventional Voigt, indicative of a better fit. It is noteworthy to remind that $\tilde{\chi}^{2}$ is a statistic parameter indicating the quality of the fit for a given protocol with different degrees of freedom (>300 for each acquired C 1s survey). For this reason, a simple comparison can be only qualitative when the calculated values amount to very few units.

A more systematically approach regards the analysis of the relative abundance of the functional groups using the two protocols and as well as the comparison with the $\mathrm{O} / \mathrm{C}$ values obtained using the area method. In general, using the conventional symmetric fit, the functional group C 1s intensities obtained were systematically higher, because the symmetric fit had to compensate for the long tail of the asymmetric $\mathrm{sp}^{2}$ peak.

The O/C values calculated by the two fitting protocols were then compared with those calculated with the area method.

The conventional symmetric fit provided an $\mathrm{O} / \mathrm{C}_{\text {fit }}$ ratio of $0.15 \pm 0.01$, twice the $\mathrm{O} / \mathrm{C}_{\text {area }}$ ratio $(0.069 \pm 0.001)$ whereas the APV function gave an $\mathrm{O} / \mathrm{C}_{\text {fit }}=0.08 \pm 0.01$, comparable to the $\mathrm{O} / \mathrm{C}$ area value [36].

A similar difference was observed also for more defective materials, such as RGO samples. Figure 4 shows, for example, the $\mathrm{C} 1 \mathrm{~s}$ spectra of RGO reduced at annealing temperature $\mathrm{T}_{\mathrm{ann}}=600{ }^{\circ} \mathrm{C}$. The 
Voigt fit gave a value of $0.28 \pm 0.01$, more than twice the $\mathrm{O} / \mathrm{C}_{\text {area }}(0.107 \pm 0.002)$ value, while the APV fit gave a value of $0.12 \pm 0.01$, close to the $\mathrm{O} / \mathrm{C}_{\text {area }}$ value.

We also compared the FWHM of the $\mathrm{C}$ 1s peak, measured for the different samples since variations are common in many graphitic-like materials (graphite, Kish graphite and thermally treated graphite) and depend on the degree of crystallinity and the number of defects [31].

The FWHM of multilayer CVD-G (i.e. more than 30 single layers of graphene grown on nickel [36]) was $0.83 \pm 0.05$, in excellent agreement to that of the HOPG used as a model for the $\mathrm{sp}^{2}$ function $(0.82 \pm 0.02)$.

The $\mathrm{sp}^{2}$ peak width (FWHM) of the RGO annealed at $600^{\circ} \mathrm{C}$ obtained with the APV fit was $1.20 \pm 0.03$ $\mathrm{eV}$, in good agreement with the values measured on disordered carbon structures such as Kish graphite [52] whereas samples of more reduced RGO $\left(\mathrm{T}_{\mathrm{ann}}=900^{\circ} \mathrm{C}\right)$ gave a smaller $\mathrm{FWHM}=$ $1.10 \pm 0.03$ (figure S3).
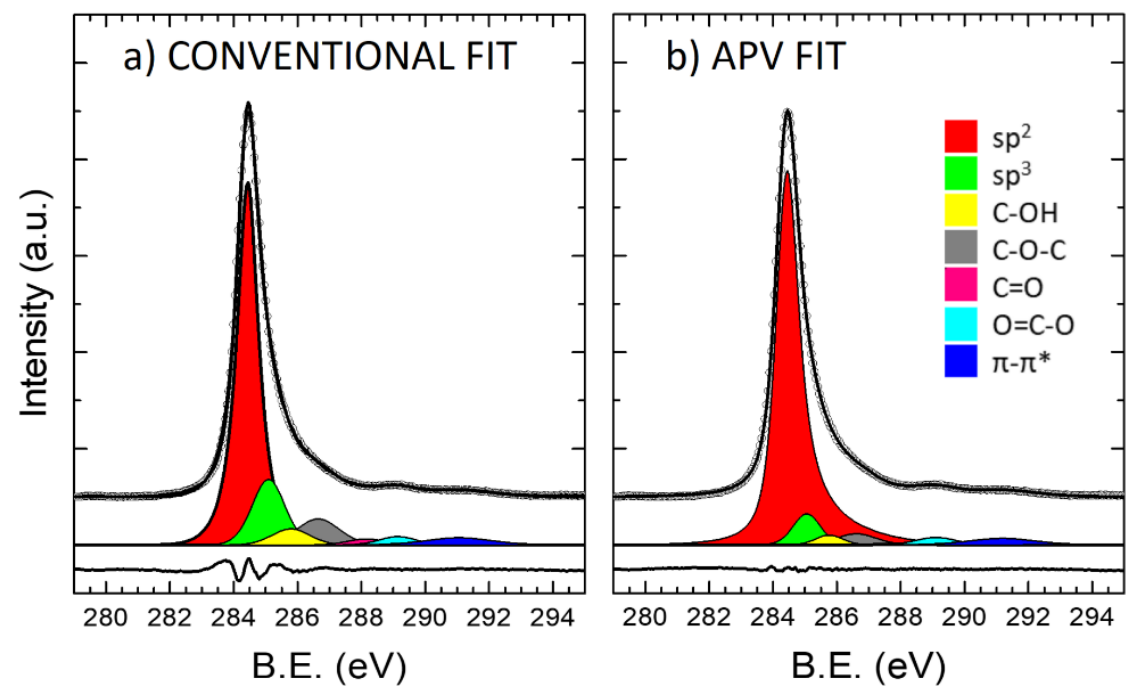

\begin{tabular}{|c|c|c|c|c|c|c|c|}
\hline c) & \multicolumn{6}{|c|}{ Relative concentration (\%) } & \\
\hline Model & $\mathrm{sp}^{2}$ & $\mathrm{sp}^{3}$ & $\mathrm{C}-\mathrm{OH}$ & $\mathrm{C}-\mathrm{O}-\mathrm{C}$ & $C=O$ & $\mathrm{O}-\mathrm{C}=\mathrm{O}$ & $\mathrm{O} / \mathrm{C}_{\text {fit }}$ \\
\hline $\begin{array}{l}\text { Conventional } \\
\text { symmetric fit }\end{array}$ & $67.6 \pm 0.9$ & $0.7 \pm 0.2$ & $14.6 \pm 0.5$ & $11.3 \pm 0.2$ & $3.5 \pm 0.1$ & $2.3 \pm 0.1$ & $0.15 \pm 0.01$ \\
\hline APV fit & $87.2 \pm 0.9$ & $<0.2$ & $5.4 \pm 0.1$ & $4.3 \pm 0.2$ & $1.8 \pm 0.1$ & $1.3 \pm 0.1$ & $0.08 \pm 0.01$ \\
\hline
\end{tabular}

Fig. 3. C 1s spectra of CVD-G grown on $\mathrm{Ni}$, measured after etching the $\mathrm{Ni}$. a) Conventional symmetric Voigt fit; b) APV fit. Shirley background was subtracted and an offset added. Note that in the conventional symmetric fit the functional group intensities look systematically higher, because the symmetric fit had to compensate for the long tail of the asymmetric $\mathrm{sp}^{2}$ peak. The $\mathrm{O} / \mathrm{C}_{\text {area }}$ calculated using oxygen and carbon peaks was $0.069 \pm 0.001$. The table reports the precise abundancy (in \%) of the different chemical species, obtained with the two fitting protocols. 

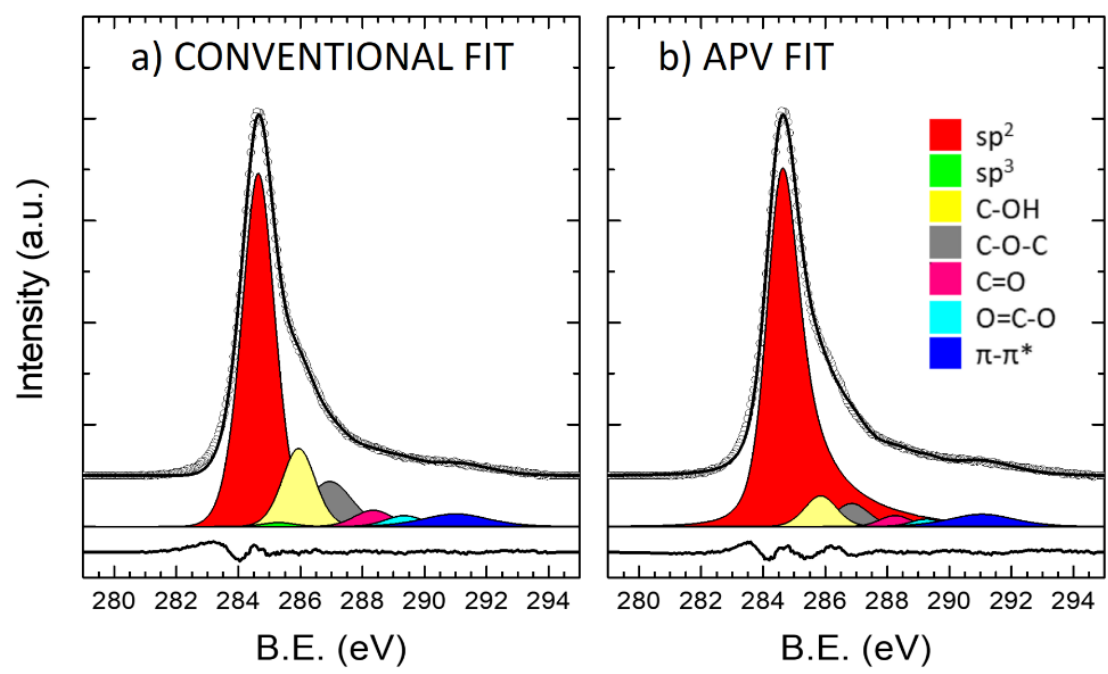

\begin{tabular}{|c|c|c|c|c|c|c|c|}
\hline \multirow{2}{*}{ C) } & \multicolumn{6}{|c|}{ Relative concentration (\%) } & \\
\hline & $\mathrm{sp}^{2}$ & $\mathrm{sp}^{3}$ & $\mathrm{C}-\mathrm{OH}$ & $\mathrm{C}-\mathrm{O}-\mathrm{C}$ & $\mathrm{C}=\mathrm{O}$ & $\mathrm{O}-\mathrm{C}=\mathrm{O}$ & $\mathrm{O} / \mathrm{C}_{\text {fit }}$ \\
\hline $\begin{array}{l}\text { Conventional } \\
\text { symmetric fit }\end{array}$ & $67.6 \pm 0.9$ & $0.7 \pm 0.2$ & $14.6 \pm 0.5$ & $11.3 \pm 0.2$ & $3.5 \pm 0.1$ & $2.3 \pm 0.1$ & $0.28 \pm 0.01$ \\
\hline APV fit & $87.2 \pm 0.9$ & $<0.2$ & $5.4 \pm 0.1$ & $4.3 \pm 0.2$ & $1.8 \pm 0.1$ & $1.3 \pm 0.1$ & $0.12 \pm 0.01$ \\
\hline
\end{tabular}

Fig. 4. C 1s spectra of RGO $\left(\mathrm{T}_{\mathrm{ann}}=600^{\circ} \mathrm{C}\right)$. a) Conventional symmetric Voigt fit; b) APV fit. Shirley background was subtracted and an offset added. The $\mathrm{O} / \mathrm{C}_{\text {area }}$ was $0.107 \pm 0.002$. The table reports the precise abundancy (in \%) of the different chemical species, obtained with the two fitting protocols.

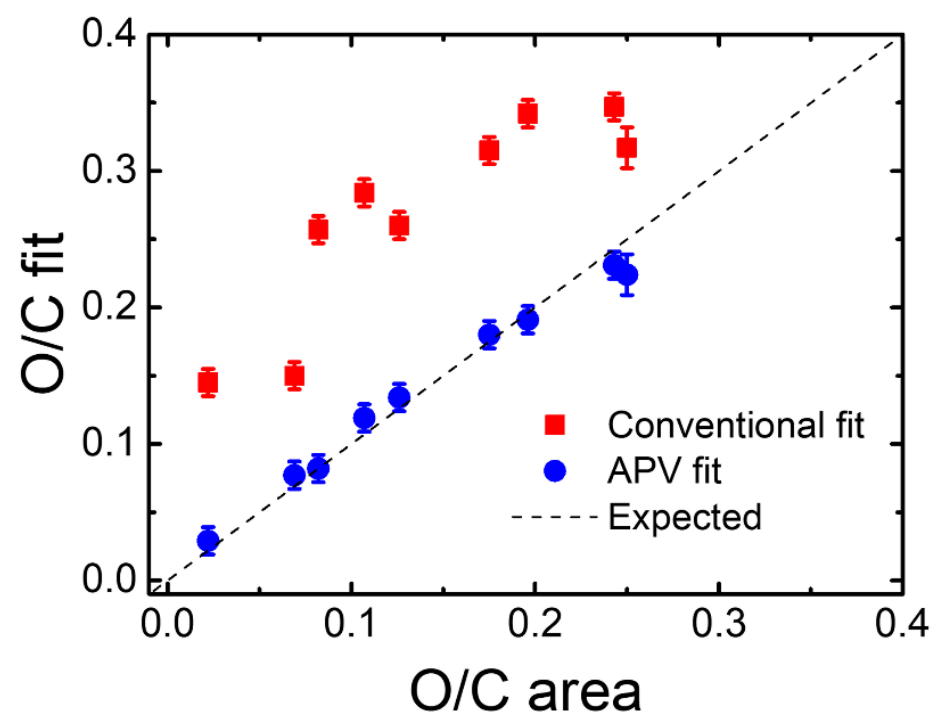

Fig. 5. Correlation plot: $\mathrm{O} / \mathrm{C}_{\text {fit }}$ vs. O/Carea. Values obtained from analysis of XPS data from our samples using the conventional symmetric model ( $\square$ ), as in the standard literature approach, and the APV fit $(\bullet)$ developed here. The dotted line corresponds to perfect, ideal correlation. 
Similar analyses were performed on nine different GRMs and the results are reported in the correlation plot (figure 5). All values are tabulated in tables S3, S4 and S5 in the SI. While conventional symmetric fitted data points are scattered on the graph, O/C values calculated with the APV fit lie very close to the bisector line, providing straightforward evidence of the accuracy of the developed APV protocol. The reduced chi-squared value obtained with the APV fit $\left(\tilde{\chi}^{2}=1.2\right)$, was significantly better than that obtained using the conventional symmetric Voigt fit $\left(\tilde{\chi}^{2}=184\right)$ [51], which was affected by a systematic overestimation of the oxygen content.

The APV protocol was then used for more challenging samples, such as GRM thin films deposited on substrates containing oxygen (silicon with native oxide) (figure 6).
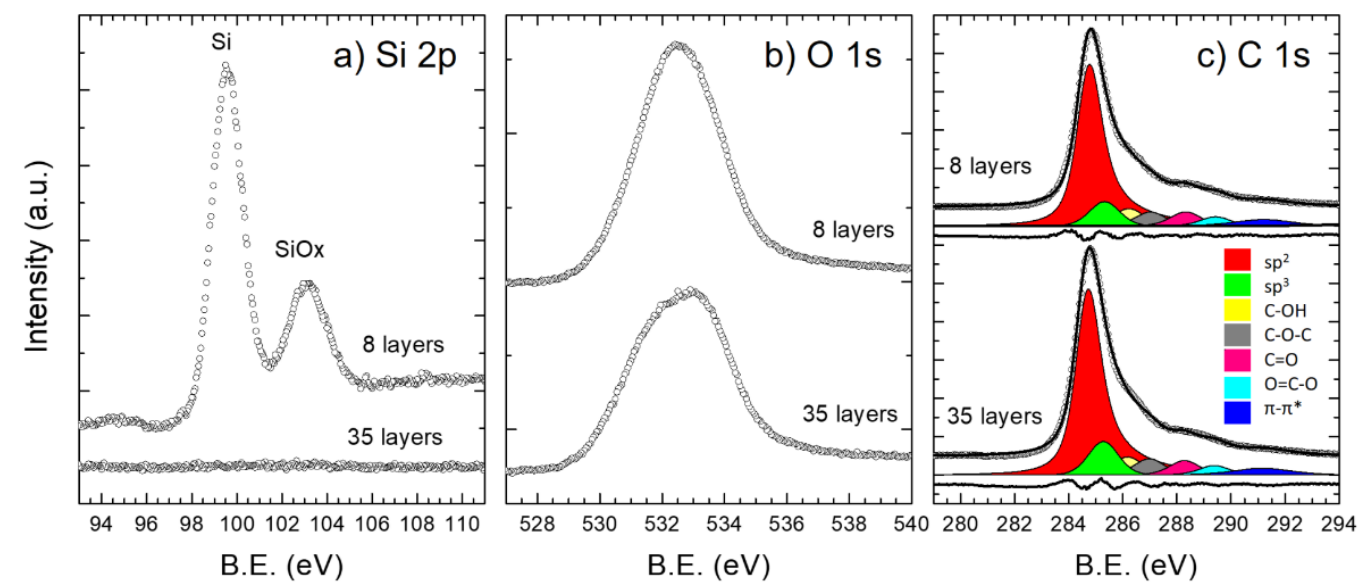

Fig. 6. XPS spectra a) Si 2p, b) O 1s and c) APV deconvolution of C 1 s of the RGO $\left(\mathrm{T}_{\mathrm{ann}}=300^{\circ} \mathrm{C}\right)$ with two thicknesses: $8 \pm 1\left(\mathrm{O} / \mathrm{C}_{\mathrm{fit}}=0.20 \pm 0.01\right)$ and $35 \pm 2\left(\mathrm{O} / \mathrm{C}_{\mathrm{fit}}=0.18 \pm 0.01\right)$ layers. The Si $2 \mathrm{p}$ signal was not detectable on the 35-layer films.

We calculated the $\mathrm{O} / \mathrm{C}_{\text {fit }}$ ratio of thick (35 layers) and thin (8 layers) of RGO coatings, each of them annealed at three different temperatures $\left(900,700\right.$ and $300{ }^{\circ} \mathrm{C}$ ). The thicker sample gave good agreement between $\mathrm{O} / \mathrm{C}_{\text {fit }}$ and $\mathrm{O} / \mathrm{C}_{\text {area }}$ with no $\mathrm{Si} 2 \mathrm{p}$ contribution; conversely, the XPS of the thin film clearly showed the Si $2 \mathrm{p}$ signal from the underlying substrate (figure $6 \mathrm{a}$ ). It was therefore not possible to estimate the $\mathrm{O} / \mathrm{C}_{\text {area }}$ of the 8-layer sample, because the $\mathrm{O} 1 \mathrm{~s}$ signal (figure $6 \mathrm{~b}$ ) showed contributions from both the substrate $\left(\mathrm{SiO}_{\mathrm{x}}\right)$ and the film (C-O functional groups). Our APV fitting protocol (Figure 6c) of the 8-layer sample was thus the only valid way to estimate the degree of RGO oxidation from the XPS data. The APV fit was performed on data from samples with three different degrees of oxidation obtained at three different annealing temperatures $\left(\mathrm{T}_{\mathrm{ann}}=300,600\right.$ and $\left.900^{\circ} \mathrm{C}\right)$, the results being summarized in table 1 . The corresponding calculated $\mathrm{O} / \mathrm{C}_{\text {fit }}$ ratio depends on the annealing temperature, as expected, due to the reduction of GO, but not on the thickness of the film, confirming 
that it indicates an intrinsic property of the material, with no interference from the substrate nor contamination.

\begin{tabular}{ccc}
\hline \multirow{2}{*}{$\begin{array}{c}\text { Annealing } \\
\text { temperature }\left({ }^{\circ} \mathrm{C}\right)\end{array}$} & \multicolumn{2}{c}{$\mathrm{O} / \mathrm{C}_{\mathrm{fit}}$, as calculated with APV } \\
\cline { 2 - 3 } & $35 \pm 2$ layers & $8 \pm 1$ layers \\
\hline 900 & $0.03 \pm 0.01$ & $0.02 \pm 0.01$ \\
700 & $0.08 \pm 0.01$ & $0.09 \pm 0.01$ \\
300 & $0.18 \pm 0.01$ & $0.20 \pm 0.01$
\end{tabular}

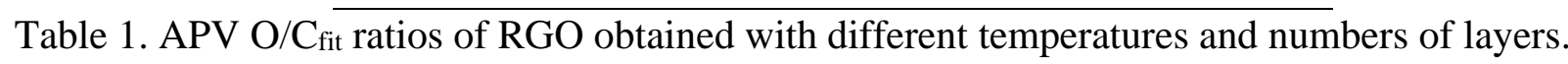

After testing the general validity of our APV fit, we applied it to the published data shown in figure 2. Figure 7 shows the calculated $\mathrm{O} / \mathrm{C}$ values in the correlation plot. Our analysis is based on data extracted using the Matlab Grabit routine from the pdf files, and thus with lower signal-to-noise ratios than in the original publications. However, even with these limitations, one can see much better agreement between the estimated values of $\mathrm{O} / \mathrm{C}_{\text {area }}$ and $\mathrm{O} / \mathrm{C}_{\text {fit }}$, (Figure $7, \tilde{\chi}^{2}=5.0$ ) compared to the values reported in the original articles (Figure 2, $\tilde{\chi}^{2}=29$ ). In general, the agreement was good for O/C $<0.15$, but less so for more complex, highly oxidized samples. At high oxygen content the C $1 \mathrm{~s}$ spectrum is more structured, giving a much lower signal-to-noise ratio. For example, we obtained perfect agreement between $\mathrm{O} / \mathrm{C}_{\text {area }}$ and $\mathrm{O} / \mathrm{C}_{\text {fit }}$ for two samples of Mattevi et al. with low oxygen content (green triangles) [15], whereas the third, more oxidized sample in the same article did not lie so close to the graph bisector.

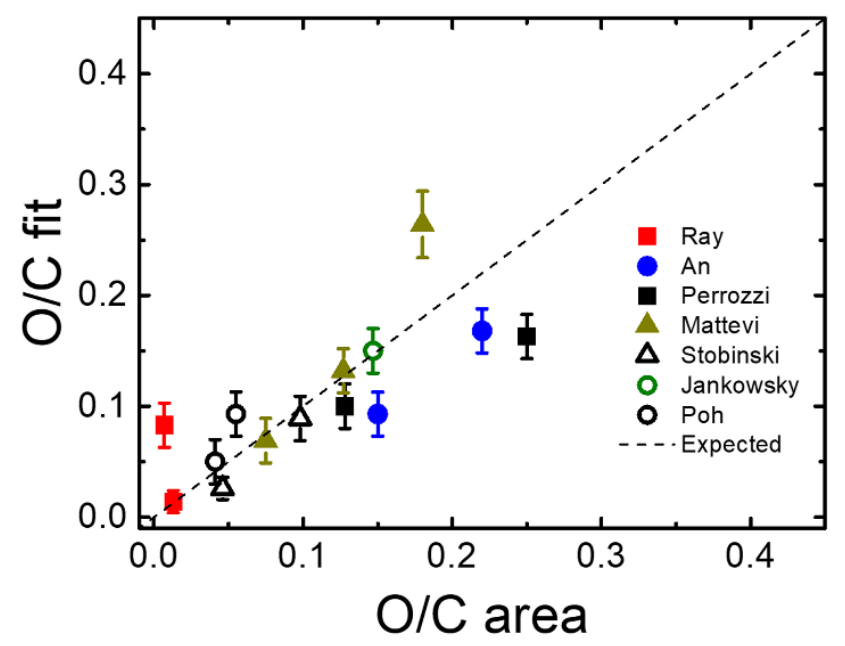

Fig. 7. Correlation plot $\mathrm{O} / \mathrm{C}_{\text {area }}$ vs $\mathrm{O} / \mathrm{C}_{\text {fit. }}$ of previously published data. $\mathrm{O} / \mathrm{C}_{\text {fit }}$ calculated after the digitalization of published data with APV fit. Source of data: Ray [21]; An [22]; Perrozzi [20]; Mattevi [17]; Stobinski [16]; Jankowski [19]; Poh [18]. 


\section{Conclusions}

The APV protocol described here allows much greater accuracy than conventional protocols in estimating by XPS the O/C ratio and the carbon functionalization of materials.

As solid-state physics has shown, the $\mathrm{sp}^{2} \mathrm{C} 1 \mathrm{~s}$ contribution to the XPS signal is strongly asymmetric, but this fact has often not been taken into account in quantitative XPS analysis leading to an overestimation of the oxygen content and a wrong chemical analysis. Our approach thus allows one to eliminate oversimplifications commonly used in the XPS analysis of GRMs and develop a more accurate and quite universal approach in the analysis of the XPS data.

The APV fit gives results based only on the $\mathrm{C} 1 \mathrm{~s}$ XPS signal, thus avoiding artefacts from $\mathrm{O}$ 1s signals from oxygen contamination of the samples or from the substrate. Consequently, this allows XPS analysis and $\mathrm{O} / \mathrm{C}$ ratio estimation over a much wider variety of samples compared to the usual methods, based on the ratio of carbon and oxygen XPS peak areas. This is particularly important, for example, in the characterization of materials for electronics or energy storage, where graphene is often combined with silicon or other metal oxides.

The new APV protocol was verified on nine groups of prepared GRM samples with widely varying oxygen content, thickness and substrate, giving results corresponding closely to the nature of those samples.

APV also provides a valid contribution to improving the analysis of previously published XPS data enhancing the value of past work in the field. For all the XPS data presented here as well as for the selected previously published data, APV showed more coherent results than conventional protocols. Finally, our approach can be easily applied not only to GRMs, but also to XPS data on other carbonbased materials, providing a much clearer picture of their nature and quality.

\section{Acknowledgements}

The research leading to these results received funding from the European Union Horizon 2020 research and innovation programme under GrapheneCore2 785219 - Graphene Flagship and and the EC Marie-Curie ITN- iSwitch (GA no. 642196). The XPS instrument was purchased with financial support from the Emilia-Romagna (Italy) regional project POR FES 2007-2013. The authors are particularly grateful to Zhenyuan Xia for providing the EGO samples, to Meganne Christian for the CVD-Graphene samples, to Rita Rizzoli and Rita Tonini for the use of the HV oven for the heat treatment. 


\section{References}

[1] V. Palermo, Not a molecule, not a polymer, not a substrate ... the many faces of graphene as a chemical platform, Chemical Communications 49(28) (2013) 2848-2857, 10.1039/c3cc37474b

[2] K. Kouroupis-Agalou, A. Liscio, E. Treossi, L. Ortolani, V. Morandi, N.M. Pugno, et al., Fragmentation and exfoliation of 2-dimensional materials: a statistical approach, Nanoscale 6(11) (2014) 5926-5933,

$10.1039 / \mathrm{c} 3 \mathrm{nr} 06919 \mathrm{~b}$

[3] J. Wei, Z. Zang, Y. Zhang, M. Wang, J. Du, X. Tang, Enhanced performance of light-controlled conductive switching in hybrid cuprous oxide/reduced graphene oxide (Cu2O/rGO) nanocomposites, Opt. Lett. 42(5) (2017) 911-914, 10.1364/OL.42.000911

[4] V. Palermo, I.A. Kinloch, S. Ligi, N.M. Pugno, Nanoscale Mechanics of Graphene and Graphene Oxide in Composites: A Scientific and Technological Perspective, Advanced Materials 28(29) (2016) 6232-6238, 10.1002/adma.201505469

[5] C.A. Amadei, P. Arribas, C.D. Vecitis, Graphene oxide standardization and classification: Methods to support the leap from lab to industry, Carbon 133 (2018) 398-409,

https://doi.org/10.1016/j.carbon.2018.02.091

[6] P. Wick, A.E. Louw-Gaume, M. Kucki, H.F. Krug, K. Kostarelos, B. Fadeel, et al., Classification Framework for Graphene-Based Materials, Angewandte Chemie International Edition 53(30) (2014) 7714-7718, 10.1002/anie.201403335

[7] A. Bianco, H.-M. Cheng, T. Enoki, Y. Gogotsi, R.H. Hurt, N. Koratkar, et al., All in the graphene family - A recommended nomenclature for two-dimensional carbon materials, Carbon 65 (2013) 1-6, 10.1016/j.carbon.2013.08.038

[8] A. Liscio, K. Kouroupis-Agalou, X.D. Betriu, A. Kovtun, E. Treossi, N.M. Pugno, et al., Evolution of the size and shape of 2D nanosheets during ultrasonic fragmentation, 2d Materials 4(2) (2017), 10.1088/2053$1583 / \mathrm{aa} 57 \mathrm{ff}$

[9] A.C. Ferrari, Raman spectroscopy of graphene and graphite: Disorder, electron-phonon coupling, doping and nonadiabatic effects, Solid State Communications 143(1-2) (2007) 47-57, 10.1016/j.ssc.2007.03.052 [10] R. Larciprete, S. Fabris, T. Sun, P. Lacovig, A. Baraldi, S. Lizzit, Dual Path Mechanism in the Thermal Reduction of Graphene Oxide, Journal of the American Chemical Society 133(43) (2011) 17315-17321, 10.1021/ja205168x

[11] F. Buchner, K. Forster-Tonigold, M. Bozorgchenani, A. Gross, R.J.r. Behm, Interaction of a selfassembled ionic liquid layer with graphite (0001): A combined experimental and theoretical study, The journal of physical chemistry letters $7(2)$ (2016) 226-233,

[12] R.J. Zaldivar, P.M. Adams, J. Nokes, H.I. Kim, Surface functionalization of graphenelike materials by carbon monoxide atmospheric plasma treatment for improved wetting without structural degradation, Journal of Vacuum Science \& Technology B, Nanotechnology and Microelectronics: Materials, Processing, Measurement, and Phenomena 30(3) (2012) 03D107, 10.1116/1.3695337

[13] S. Rosenzweig, G.A. Sorial, E. Sahle-Demessie, D.C. McAvoy, Optimizing the physical-chemical properties of carbon nanotubes (CNT) and graphene nanoplatelets (GNP) on Cu(II) adsorption, Journal of Hazardous Materials 279 (2014) 410-417, http://dx.doi.org/10.1016/i.jhazmat.2014.07.015

[14] G. Maccaferri, C. Zanardi, Z.Y. Xia, A. Kovtun, A. Liscio, F. Terzi, et al., Systematic study of the correlation between surface chemistry, conductivity and electrocatalytic properties of graphene oxide nanosheets, Carbon 120 (2017) 165-175, 10.1016/j.carbon.2017.05.030

[15] D. Posener, The shape of spectral lines: Tables of the Voigt profile, Australian Journal of Physics 12(2) (1959) 184-196,

[16] L. Stobinski, B. Lesiak, A. Malolepszy, M. Mazurkiewicz, B. Mierzwa, J. Zemek, et al., Graphene oxide and reduced graphene oxide studied by the XRD, TEM and electron spectroscopy methods, Journal of Electron Spectroscopy and Related Phenomena 195 (2014) 145-154,

http://dx.doi.org/10.1016/j.elspec.2014.07.003 
[17] C. Mattevi, G. Eda, S. Agnoli, S. Miller, K.A. Mkhoyan, O. Celik, et al., Evolution of Electrical, Chemical, and Structural Properties of Transparent and Conducting Chemically Derived Graphene Thin Films, Advanced Functional Materials 19(16) (2009) 2577-2583, 10.1002/adfm.200900166

[18] H.L. Poh, F. Sanek, A. Ambrosi, G.J. Zhao, Z. Sofer, M. Pumera, Graphenes prepared by Staudenmaier, Hofmann and Hummers methods with consequent thermal exfoliation exhibit very different electrochemical properties, Nanoscale 4(11) (2012) 3515-3522, 10.1039/c2nr30490b

[19] O. Jankovsky, P. Simek, M. Novacek, J. Luxa, D. Sedmidubsky, M. Pumera, et al., Use of deuterium labelling-evidence of graphene hydrogenation by reduction of graphite oxide using aluminium in sodium hydroxide, RSC Advances 5(24) (2015) 18733-18739, http://dx.doi.org/10.1039/C4RA16794E

[20] F. Perrozzi, S. Prezioso, M. Donarelli, F. Bisti, P. De Marco, S. Santucci, et al., Use of Optical Contrast To Estimate the Degree of Reduction of Graphene Oxide, Journal of Physical Chemistry C 117(1) (2013) 620625, 10.1021/jp3069738

[21] A.K. Ray, R.K. Sahu, V. Rajinikanth, H. Bapari, M. Ghosh, P. Paul, Preparation and characterization of graphene and $\mathrm{Ni}$-decorated graphene using flower petals as the precursor material, Carbon 50(11) (2012) 4123-4129, 10.1016/j.carbon.2012.04.060

[22] Q. An, A.N. Rider, E.T. Thostenson, Electrophoretic deposition of carbon nanotubes onto carbon-fiber fabric for production of carbon/epoxy composites with improved mechanical properties, Carbon 50(11) (2012) 4130-4143, http://dx.doi.org/10.1016/i.carbon.2012.04.061

[23] R.S. Hidalgo, D. López-Díaz, M.M. Velázquez, Graphene Oxide Thin Films: Influence of Chemical Structure and Deposition Methodology, Langmuir 31(9) (2015) 2697-2705, 10.1021/la5029178

[24] D. Joung, S.I. Khondaker, Efros-Shklovskii variable-range hopping in reduced graphene oxide sheets of varying carbon $\$ s\{p\}^{\wedge}\{2\} \$$ fraction, Physical Review B 86(23) (2012) 235423,

[25] R. Jayasingha, A. Sherehiy, S.-Y. Wu, G.U. Sumanasekera, In Situ Study of Hydrogenation of Graphene and New Phases of Localization between Metal-Insulator Transitions, Nano Letters 13(11) (2013) 5098-

$5105,10.1021 / \mathrm{n} \mid 402272 \mathrm{~b}$

[26] M. Copuroglu, P. Aydogan, E.O. Polat, C. Kocabas, S. Süzer, Gate-Tunable Photoemission from Graphene Transistors, Nano Letters 14(5) (2014) 2837-2842, 10.1021/nl500842y

[27] S. Kim, S. Shin, T. Kim, H. Du, M. Song, C. Lee, et al., Robust graphene wet transfer process through low molecular weight polymethylmethacrylate, Carbon 98 (2016) 352-357,

http://dx.doi.org/10.1016/i.carbon.2015.11.027

[28] Z.-S. Wu, W. Ren, L. Gao, B. Liu, C. Jiang, H.-M. Cheng, Synthesis of high-quality graphene with a predetermined number of layers, Carbon 47(2) (2009) 493-499, https://doi.org/10.1016/j.carbon.2008.10.031 [29] S. Yumitori, Correlation of C-1s chemical state intensities with the O-1s intensity in the XPS analysis of anodically oxidized glass-like carbon samples, Journal of Materials Science 35(1) (2000) 139-146, 10.1023/a:1004761103919

[30] S. Hufner, Photoelectron Spectroscopy: Principles and Applications, 2003.

[31] H. Estrade-Szwarckopf, XPS photoemission in carbonaceous materials: A "defect" peak beside the graphitic asymmetric peak, Carbon 42(8-9) (2004) 1713-1721, 10.1016/j.carbon.2004.03.005

[32] K.C. Prince, I. Ulrych, M. Peloi, B. Ressel, V. Chab, C. Crotti, et al., Core-level photoemission from graphite, Physical Review B 62(11) (2000) 6866-6868, 10.1103/PhysRevB.62.6866

[33] E. Treossi, M. Melucci, A. Liscio, M. Gazzano, P. Samorì, V. Palermo, High-Contrast Visualization of Graphene Oxide on Dye-Sensitized Glass, Quartz, and Silicon by Fluorescence Quenching, Journal of the American Chemical Society 131(43) (2009) 15576-15577, 10.1021/ja9055382

[34] A. Kovtun, 2D graphene-based materials. Interplay between composition and electrical properties., FIM, Università degli Studi di MODENA e REGGIO EMILIA, 2017.

[35] A. Liscio, G.P. Veronese, E. Treossi, F. Suriano, F. Rossella, V. Bellani, et al., Charge transport in graphene-polythiophene blends as studied by Kelvin Probe Force Microscopy and transistor characterization, Journal of Materials Chemistry 21(9) (2011) 2924-2931, 10.1039/COJM02940H

[36] M. Christian, L. Venturi, L. Ortolani, F. Liscio, R. Rizzoli, V. Palermo, et al., Synthesis of High-Density Graphene Foams Using Nanoparticle Templates, in: V. Morandi, L. Ottaviano (Eds.), GraphITA : Selected 
papers from the Workshop on Synthesis, Characterization and Technological Exploitation of Graphene and 2D Materials Beyond Graphene, Springer International Publishing, Cham, 2017, pp. 185-196.

[37] M. Batzill, The surface science of graphene: Metal interfaces, CVD synthesis, nanoribbons, chemical modifications, and defects, Surface Science Reports 67(3-4) (2012) 83-115, 10.1016/j.surfrep.2011.12.001

[38] Z.Y. Xia, G. Giambastiani, C. Christodoulou, M.V. Nardi, N. Koch, E. Treossi, et al., Synergic Exfoliation of Graphene with Organic Molecules and Inorganic lons for the Electrochemical Production of Flexible Electrodes, Chempluschem 79(3) (2014) 439-446, 10.1002/cplu.201300375

[39] D.A. Shirley, High-resolution X-ray photoemission spectrum of the valence bands of gold, Physical Review B 5(12) (1972) 4709,

[40] D. Briggs, M.P. Seah, Practical surface analysis : by auger and x-ray photoelectron spectroscopy, Wiley, Chichester; New York, 1983.

[41] F. Perrozzi, S. Croce, E. Treossi, V. Palermo, S. Santucci, G. Fioravanti, et al., Reduction dependent wetting properties of graphene oxide, Carbon 77 (2014) 473-480, 10.1016/j.carbon.2014.05.052

[42] M. Inukai, E. Ikenaga, T. Muro, S. Ogawa, Y. Ojiro, Y. Takakuwa, et al., Spectroscopic Analysis of Graphitization and Grain Orientation of Carbon Films Grown by Photoemission-Assisted Plasma-Enhanced Chemical Vapor Deposition, Japanese Journal of Applied Physics 52(6) (2013), 10.7567/jjap.52.065503

[43] J.A. Leiro, M.H. Heinonen, T. Laiho, I.G. Batirev, Core-level XPS spectra of fullerene, highly oriented pyrolitic graphite, and glassy carbon, Journal of Electron Spectroscopy and Related Phenomena 128(2-3) (2003) 205-213, 10.1016/s0368-2048(02)00284-0

[44] M. Schmid, H.P. Steinruck, J.M. Gottfried, A new asymmetric Pseudo-Voigt function for more efficient fitting of XPS lines, Surface and Interface Analysis 46(8) (2014) 505-511, 10.1002/sia.5521

[45] Y. Shvyd'ko, X-Ray Optics. High-Energy-Resolution Applications, Springer, Berlin, 2004.

[46] P.S. Bagus, E.S. Ilton, C.J. Nelin, The interpretation of XPS spectra: Insights into materials properties, Surface Science Reports 68(2) (2013) 273-304, 10.1016/j.surfrep.2013.03.001

[47] G. Beamson, A. Bunn, D. Briggs, High-resolution monochromated XPS of poly(methyl methacrylate) thin films on a conducting substrate, Surface and Interface Analysis 17(2) (1991) 105-115, http://doi.org/10.1002/sia.740170206

[48] P. Vanattekum, G.K. Wertheim, EXCITONIC EFFECTS IN CORE-HOLE SCREENING, Physical Review Letters 43(25) (1979) 1896-1898, 10.1103/PhysRevLett.43.1896

[49] D.Q. Yang, E. Sacher, Carbon 1s X-ray photoemission line shape analysis of highly oriented pyrolytic graphite: The influence of structural damage on peak asymmetry, Langmuir 22(3) (2006) 860-862, 10.1021/la052922r

[50] S. Doniach, M. Sunjic, Many-electron singularity in X-ray photoemission and X-ray line spectra from metals, Journal of Physics C: Solid State Physics 3(2) (1970) 285,

[51] J. Taylor, Introduction to error analysis, the study of uncertainties in physical measurements, 1997.

[52] T. Takahagi, A. Ishitani, XPS study on the surface structure of carbon fibers using chemical modification and C1s line shape analysis, Carbon 26(3) (1988) 389-395, https://doi.org/10.1016/0008-6223(88)90231-X 